\title{
LE DIMENSIONI DELLE SONDE RADIOATTIVE E LEFFETTO DI CARICA SPAZIALE
}

\author{
Rexato Cialdea
}

In un recente lavoro (') e sata determinata teoricamente löinfluenza della calrica spaziale -ul potenziale misurato da una sonda badivatlisa, posa in un campo elettrico generalo da due amalure piane e parallele: nel caso lateralmente indefinilo, furono trovati vatlori diversi da quelli otlenuti sperimentalmente $\left(^{2}\right)$ con una comune sonda a polonio; tale differenza puó attribuirsi al fiatto che l'azione ionizzante. crat limilata ad un ristrette volume e quindi l"influenza della rarica saziale era notevolmente minore.

La presconza di una sonda radioaltiva da luogo a due distinte perlubbazioni del campo elettrico in cui è immersa. Prima di futio, il rolume sogrgelto all'azione ionizzante si può ronsiderare come un rondutore, dato l'elevato valore dellat concentrazione degli ioni d'ambo i segni isi presenti, alterando quindi il campo elettrico: qualora il conduttore stesso potesse considerirsi lateralmente indefinito, di spes-ore f' misurato lungo le linee di forza del campo e con cariea spariale eslerna nulla, il potenziale presentereble l’andamento della -pezzata b) della fịg. l. La rella $a$ della slessa figura rappresenta orsiamente il potenziale misurato dat una sonda di spessore trascurabile ri-petto allat distanza delle armature. -empe con cariea spaziale mulla al di fuori della sonda. Lat seconda perturbazione cominc $i_{a l}$ a far senlire lat slla influenza allorché gli ioni che si trovano all'esterno del volume soggello all'azione ionizzante hamno una densib lale che lat carica spaziale non possa esere trascurata: in questo catoo il polenziale assume landamento rappresentalo dalla curva teorica c). Da fuanto ora detlo. i prevedibile the la dimensione della sonda lungo le linee di forza del campo influira principalmente -ulla lunghezza dei

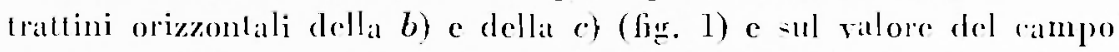
elettrivo nell’interno del condensitore; invere le altre due dimen-ioni

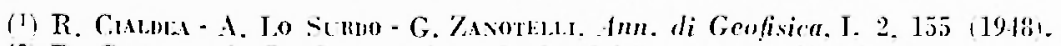

(2) R. Culdea - 1. Lo Stmo. Inn. di Geofisica. I, 1, 19 (1948). 
misurate normalmente alle linee di forza influiranno piutlosio sull'effeto della rarica spaziale. Infatti. poiche $i$ valori sperimentali oflenuli con una sonda comme ono ri-ultati più virini alla spezzala b) cle non alla ('urra $c)$, i da allendersi fhe. aumentando le dimentioni trasrersali della sonda, ei si debba arvicinare al cazo lateralmente indefunito. Consideriamo infatti la cezione del volume soggetlo allazione ionizzante, mi-urata parallelamente alle armalune del condensatore: gli ioni provenienti dalla - wa periferia segruono le linee di forza -oggetle alle perturbazioni ai bordi e quindi ranno diradando-i con ma densità che diminuisce rapidamente man mano che si allontanano; gli altri invece. provenienti dalla slla zona centrale, segurono le linec di forza non perturbale e quindi si mantengono paraileli con man densilà pressoce che costante. Di romseguenza i prevedibite che i primi diano all'offetto della cariea spaziale un contributo molto più piccolo dei secondi; mui sonda reale e fuindi funi-

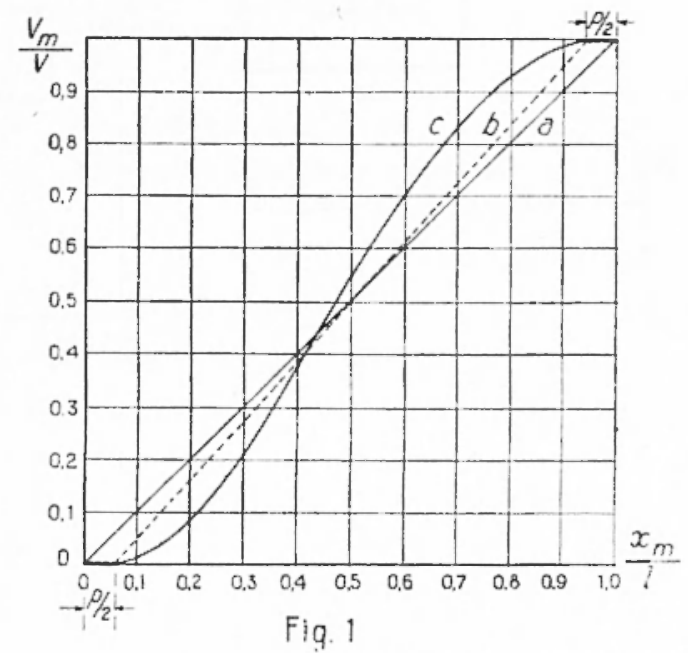
la potrà essere perciò as. cimilala alla sonda teorica indefunita allorche $i$ primi siano in nume. ro llacurabile rispeto ai secondi, cios allonche le perturbazioni ai bordi del sllo rolume soggetlo all'azione ionizzanle posimo essere rascurate.

Allo sepo dunfue di studiare l’influemal effentiva delle dimensioni e della forma di detlo rolume nei riguade dell"effello della cariea spaziale, ho condotto altre esperienze aon m numero magraiore di conde. Sia ponendole lute su una solal filat, sia distribuendole sl un telaio quadrato a maglie larghe.

Per puesta riecrea ho realizzato un metodo di mi-ura rhe mi ha

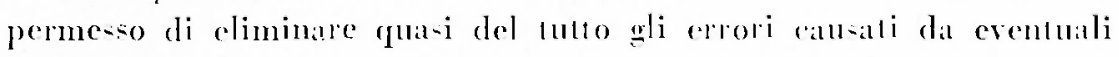


366

REbATO CALVE.

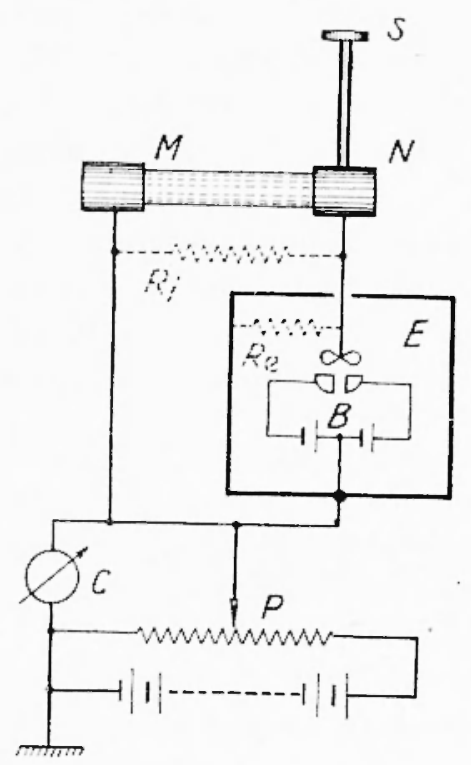

Fig. 3

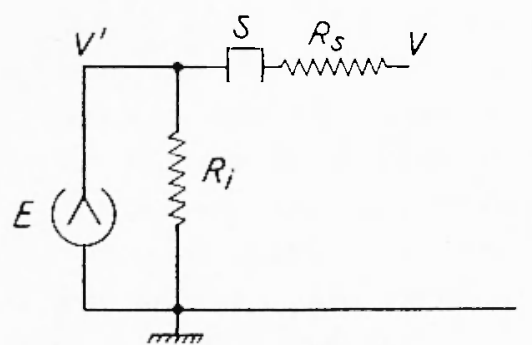

Fig. 2

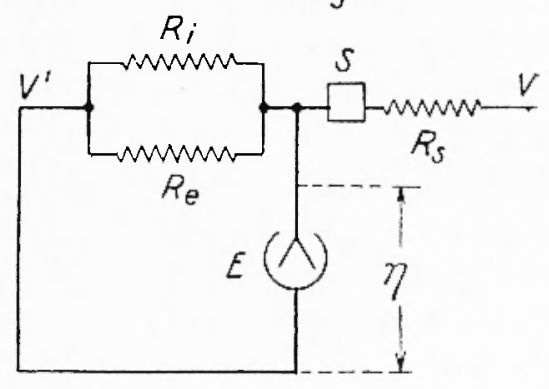

Fig. 4

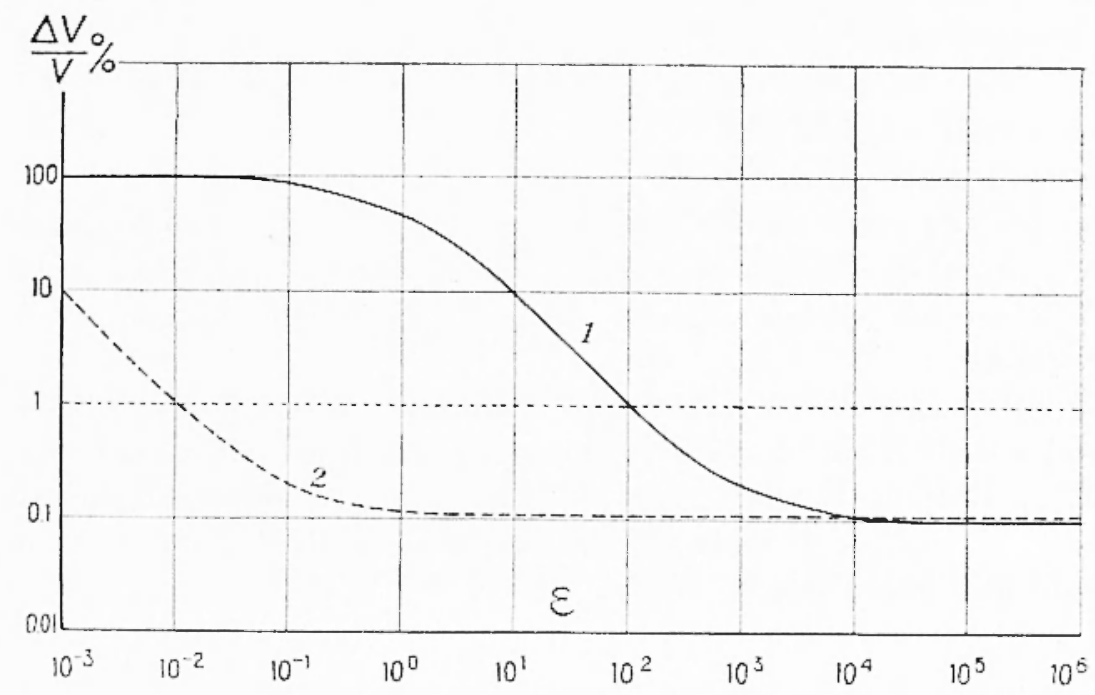

Fig. 5 
perdice dovale ad inol:mento nom perfeltu. F" nolo infitti (") whe, $\Leftrightarrow$ il -o-lewno i-olante della sonda $h_{1 i}$ mula resistenza $R_{i}$ di valore fenile. il polenziale $I^{\prime \prime}$ mi-uralo dall elelldometro ì lewalo. indicando con $R_{*}$ la resielenza apparene della sondia al potenziale $l$ del punto in r:ui e...il si trovil (fier. 2) dilla relarione

$$
1=I \frac{R_{\mathrm{i}}}{R_{\mathrm{i}}+R_{*}}=1 \frac{\varepsilon}{1+\varepsilon}
$$

dove $\mathrm{i}$ is po-lo $\varepsilon=R_{\mathrm{i}} / R_{\text {. }}$. Se ne deduer ahe lerrore relation dellat mi-lura $i$

$$
\frac{\Delta V}{T}=\frac{1}{1+\varepsilon}
$$

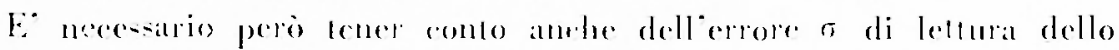
srumento nella mi-ura di polenziale con-iderata: fundi per lecrore m!a-simo romple-ivo si ha

$$
\frac{\Delta V}{V}=\frac{1}{1+\varepsilon}: \frac{\sigma}{V}
$$

Mella lig. 5 a liportato (curval 1) landamento di fuesto crerore in funzione del rapporto e nel raso: $l-100$ volt. $\sigma=10^{-1}$ volt.

Il metodo adoperalo nellit presente recerea, oltre ad ammullare quatsi del tulto le perdite per isolamento non perfello. da la po-.ibiliba di misurare il potenziale assmto dat unat sonda radioatliva mediante uno -trumento eletromagnetico a bit-si re-i-lenzal. Tale metodo consise nel porre sullizolante che so-tiene la sondas $S$ un conduttore melallico di guardia $\mathrm{N}$, che puì essere portalo a polenziali diversi mediante un sistema polenziometrieo P' (fig. 3) : quindo tale condutlore la lo slesso

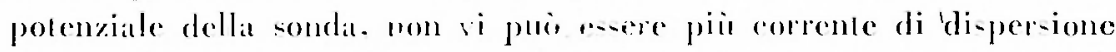
vero terra allavero il so-legno inolante posto tra $M$ ed $V$ e quindi i! -istema funziona come un i-olatore perfetlo. Per aceorgersi che $M$ ed S abbiano lo - tesso polenziale, letellrometro, montato in modo simmetrieo. ha collegato l'ago ad Ne la cusodia isolita al centro della balteria au-iliaria $B$ ed al condullore di guardia N. Anche la re-i-lenza di isolamento delleteltrometro. tra lango e la custodia, -i puó considerare

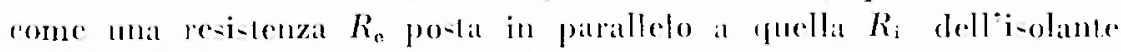

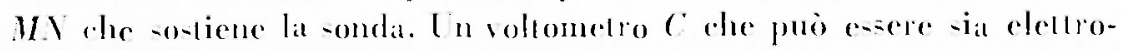

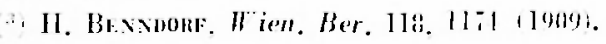


stalivo che elettromagnetico, misura il polenziale rispetto a terra di M c quindi di $S$, allorche l'elettrometro è stato riportato a zero.

Dallo schema elelrico clella firura la si può determinare quale sia l'approsimazione delle misure, quando si commeta un crore y di leltura intorno allo zero dell'elettrometro. Per determinare la differenza di potenziale esistente tra $M$ ed $N$, dovula ad una crentuale corrente di di-perione allraverso l:i=olante $M N$, occore trovare quale sia il valore massimo he può asumese tale corrente senza che l'elettrometro accusi una differenza di potenziale apprezzabile ai capi dellisolante. Sia $1 /$ la differenza di polenziale minima apprezzabile ed $i$ la corrente di dispersione, si ha

$$
\eta=i \frac{R_{i} R_{\mathrm{e}}}{R_{\mathrm{i}}+R_{\mathrm{e}}} \quad ; \quad r-V^{\prime}-\eta=i R .
$$

da queste si oltiene l'errore relativo

$$
\begin{gathered}
\frac{\Delta V^{\prime}}{Y^{\prime}}=\frac{\eta}{V} \frac{1+\varepsilon}{\varepsilon} \\
\varepsilon==\frac{R_{\mathrm{i}} R_{\mathrm{c}}}{R_{\mathrm{b}}\left(R_{\mathrm{i}}+R_{\mathrm{c}}\right)}
\end{gathered}
$$

dove si $\searrow$ po:10

tenendo inoltre conto dellerrore o di lellura dello strumento $C$ si otliene, per l'errore totale

$$
\frac{\Delta V}{V}=\frac{1+\varepsilon}{\varepsilon}+\frac{\sigma}{V}
$$

Nella fig. 5 d riportato (curva 2) l'andamento di tale errore in funzione del rupporla $\varepsilon$ e nel caso $V=100$ rolt, $\eta=10^{-2}$ roll e $\sigma-10^{-1}$ rolı.

Si vede subito dhe, mentre col primo melodo si possono ollenere delle misure eon una approsimazione delly per valori della rerisenea di isolamento rompresi lra infmilo e 100 volte quella dellat

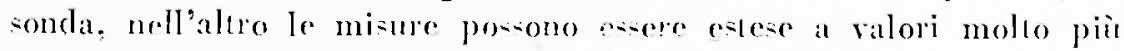
bassi della resistenza di isolamento e cios fino ad un renlesimo di quella della sonda.

Le misure sono sale aeguite ponendo le sonde in un ampo eletrice uniforme, seneralo artifieialmente mediante due grandi piani melallici, di-posi parallelamente tra loro ad una distanza di 88 cm, la $i$ quali si applicara ma differenza di polenziale. Tóelettro- 
metro atoperato era a quadranti con unı sensibiliti di $10^{-1}$ rolt/di. visione, mentre lo zero si potevil apprezzatze con unil precisione del centesimo di volt; il polenziale del conduttore di guardia renivia mi-u-

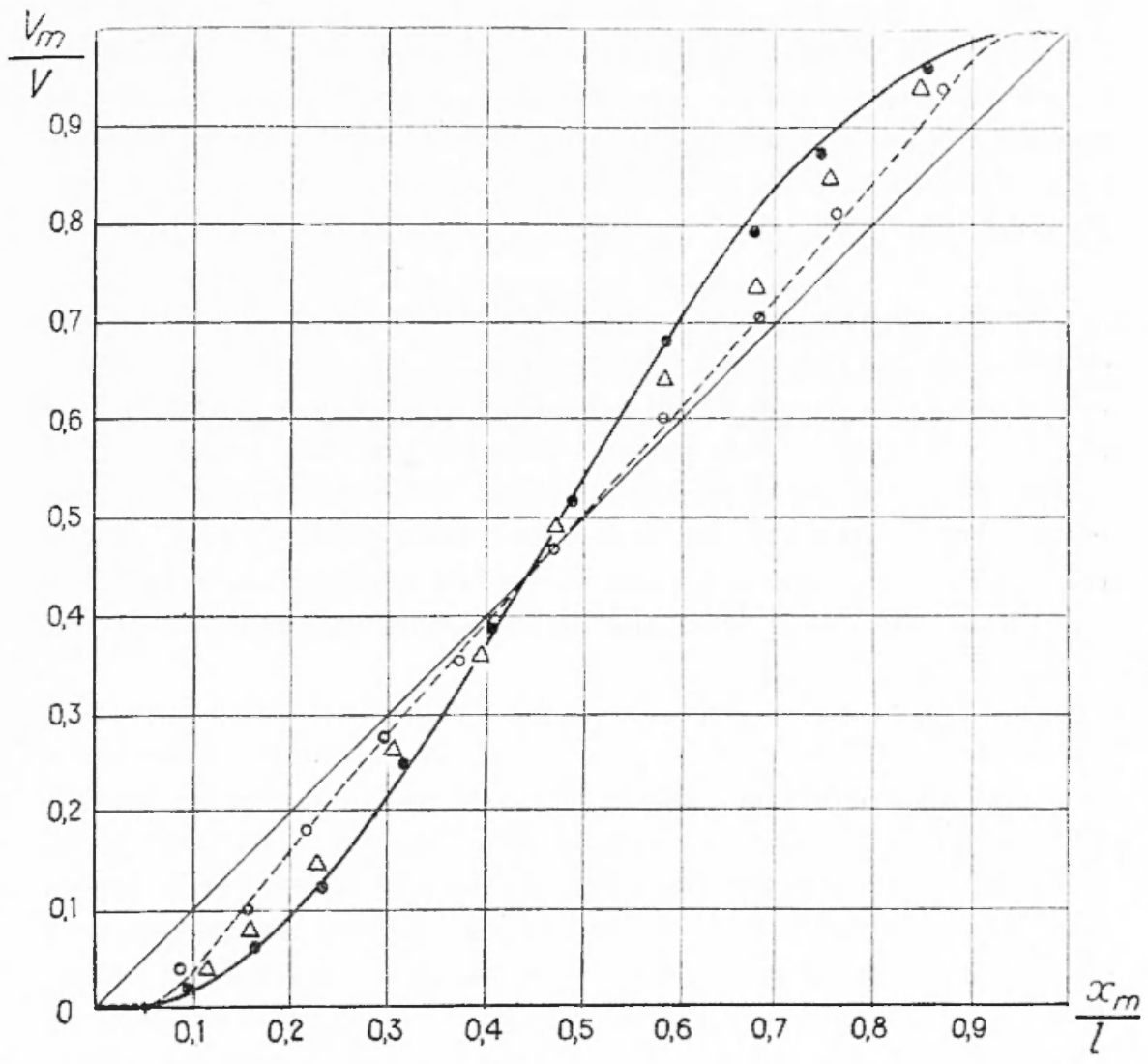

Fig. 6

ralo da in voltmetro eletromagnetico di 250.0100 ohm di re-i-lenza interna.

Conne è stalo già detlo, era da attender-i che le dimensioni della conda minuale normalmente alle linec di forza del campo. Jonero

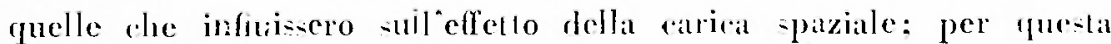
ragione ho aumentalo la sezione del volume soggelto all"agente ionizzante, parallela alle amiture del rondensilore: i rieultati -ono sati riportati nella fier. O. In queda figura sono traceiate, tral l'altro, lak relta dellandimento del potenziate nell’intemo del condensilere. in 


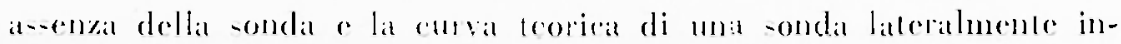
definita (curral a tratto pieno).

Sonda singola. - La -ondat contiste in un diseo metalliro di 12 mm di diametro, con una delle due falece piane ricoperta di polonio. Lo -pazio -oggello all'azione ionizzante delle partirelle at -i puo ritenere che abbia ma sezione di cirea $2.5 \mathrm{~cm}^{2}$, quando lat superficie attiva is normale alle armalture del condensatore. L'andamento del potenziale da es-a assunto è riportalo nella cuma a lrallini defla lig. 6.

Sonda renilimea. - Per aumentare le dimen-ioni, ho adoperato

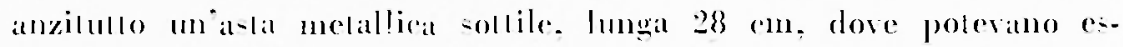
sere sistemate $2: 3$ sonde uguali alla precedente. ma a fianro dellaaltra. Tale a-ta veniva naluralmente colfocala nel campo elettrico con if lato piir lungo parallelo alle ammalure del conden-atore. La slla

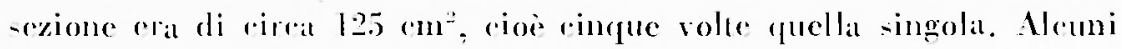
dri valori ollcmuti sono sati riporlali con dei cerchietli nella lig. $6:-i$ rede subilo che es-i s discostano di pochis-simo da quelli trovali ron la soncla singola.

Sonde quadrala. - E-sa io rostituita da mo lelaio quadratlo a due maglie entro il quale poterano essere sistemate olto sonde. Inche eso reniva rollocato nel rampo parallelamente alle amalure ded condensalore. Lal sla sezione areva una area di $125 \mathrm{~cm}^{\prime \prime}$ e le superficie attive erano di-pose in modo che nell'interno del telaio -i colende.ce dappertutto l'azione ionizante delle particelle a. Aleuni dei valori ollenuli sono ripotiali con dei triangoli nello -tesil fiğ. 6 .

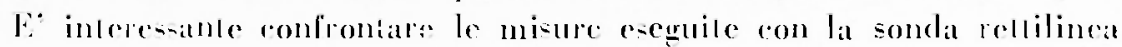
- con quellat quadrala, poiche a parita di sezione ese mo-tratno an romportamento nellamente diveros: l'effello della carioa spaziale in-

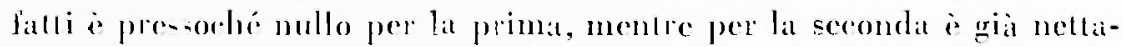
menle -ensibile. pur non raggiungendo ancora i valori leorici della -ondal laleralmente indefunila.

Grande sonda piuna. - In line ho atumenlato ancorat la sezione realizzando una conda di circa $720 \mathrm{~cm}^{2}$. A tale scopo ho adoperato me telaio a tre maglie dove potevano enere si-temate 27 sonde. Anrhe per que-so lipo, le -uperfiei altive erano di-poste in modo else lullo lo spazo inlerno del telaio fose sede dellazione ionizzante dells: 


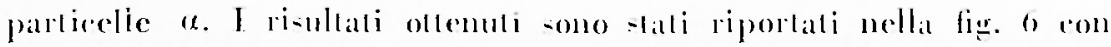
rei rechiclli pieni. Con fueste dimen-ioni si ha gia un eflello di carica spariale molto nolevole e duati prowimo a fuello leorico della -onda lilleralmente indefunita.

Roma - Istituto Vazionale di Geofisica - Maggio 1918.

\section{RIASSUNTO}

Tella presente Nola sono riportale alcune esperienze per determinare l'influenza delle dimensioni di ana sonda radioaltiva sulle fello di carica spaziale nella determinazione del potensiale eletrico: si i cosi terificala una formula looriea dhe presentara degli scarli ri. spello alle misure sperimenali eseguite con sonde comumi a polomo. 